Conclusions Vaccine injury compensation is implemented to protect the supply of vaccines and improve vaccine confidence to encourage high vaccination rates among the general population. A secondary objective is to provide timely access to compensation in the event of injury from a vaccine. Schemes that have removed the need to prove negligence and apply a low standard of proof provide timely access to compensation; these schemes also reduce administrative, legal and overhead costs and improves relations between claimants and the medical profession. Schemes that apply a higher standard of proof and enact a cumbersome claim handling and adjudication process delay timely access to compensation and reduce the number of awards. Schemes vary in the level of public awareness and support that they enjoy.

\section{SHOULD THE GOVERNMENTS FUND ASSISTED REPRODUCTIVE TECHNOLOGIES?}

Gerald O'Nolan, Jean Long, Martin Keane, Louise Farragher. Health Research Board, Dublin, Ireland

\subsection{6/bmjebm-2019-EBMLive.15}

Objectives Access to assisted reproductive technologies (ART) for those seeking treatment is dependent on their ability to afford such treatments. A combination of out-of-pocket payment, public funding and the availability of health insurance determines the uptake of ART services in most countries. The variation between countries ranges from public funding with some limitations and/or small out-of pocket payments (Australia, Belgium, France, Slovakia, Slovenia, Spain and Israel) to no funding of any kind (Malta, Switzerland and the USA).

In Ireland, such technologies have not yet been subject to government regulation or funding. The Irish Government requested the Health Research Board to investigate the costs and benefits associated with the public funding of ART for the funder, provider, and service user.

Method The methods employed to undertake this work followed the principles of a systematic review, including searching, screening, applying inclusion/exclusion criteria, data extraction, quality appraisal, and synthesis.

Results A benefit to public funding for ART in the literature reviewed includes improving access to treatment by reducing out-of-pocket payments.

Clinical benefits can also reduce the pressure on public spending. In some countries, public funding is contingent on patients and clinicians agreeing to restrict the number of embryos transferred in one cycle: single embryo transfer (SET). Where SET has occurred, there has been a significant reduction in multiple pregnancies without causing a decrease in cumulative pregnancy rates, as well savings to the public health system.

The literature outlines inferred benefits to wider society when public funding for ART is approved. In some countries, ART is seen as a social investment towards arresting the declining fertility rate, and overall future revenue receipts. Research indicates for women aged over 40 years, live births are substantially less likely following ART treatments and the financial cost of achieving a live birth is substantially more.

Conclusions Every country has a different approach to ART and the likely funding mechanism may emerge through trial and error. State funding with regulation can provide a cost- effective solution for patients who are subfertile and for wider society.

\section{8 AN INTERNATIONAL NETWORK FOR EVIDENCE-BASED RESEARCH: INTRODUCING THE EVBRES INITIATIVE}

${ }^{1}$ Jong-Wook Ban, ${ }^{2}$ Karen Robinson, ${ }^{3}$ Jennifer Yost, ${ }^{4}$ Caroline Blaine, ${ }^{5}$ Donna Ciliska, ${ }^{6}$ Klara Brunnhuber, ${ }^{7,8}$ Carsten Juhl, ${ }^{9,10}$ Robin Christensen, ${ }^{11}$ Hans Lund. ${ }^{1}$ Evidence-Based Health Care Programme, University of Oxford, Oxford, UK; ${ }^{2}$ Johns Hopkins University School of Medicine, Baltimore, USA; ${ }^{3}$ M. Louise Fitzpatrick College of Nursing, Villanova University, Villanova, USA; ${ }^{4}$ EVBRES, Western Norway University of Applied Sciences, Bergen, Norway; ${ }^{5}$ School of Nursing, McMaster University, Hamilton, Canada; ${ }^{6}$ Clinical Solutions, Elsevier Ltd., London, UK; ${ }^{7}$ Institute of Sport Science and Clinical Biomechanics, University of Southern Denmark, Odense, Denmark; ${ }^{8}$ Department of Physical and Occupational Therapy, Herlev and Gentofte Hospital, University of Copenhagen, Hellerup, Denmark ${ }^{9}$ Musculoskeletal Statistics Unit, The Parker Institute and Department of Clinical Research, SDU, Odense, Denmark; ${ }^{10}$ Department of Rheumatology, Odense University Hospital, Odense, Denmark; ${ }^{11}$ Centre for Evidence-Based Practice, Western Norway University of Applied Sciences, Bergen, Norway

\subsection{6/bmjebm-2019-EBMLive. 16}

Objectives Research on research has shown that many redundant studies would have been avoided if a systematic review has been conducted prior to starting the new study. These apparently wasteful studies limit funding available for truly important and relevant research, diminish the public's trust in research, and are unethical. Researchers planning a new study should therefore systematically review existing evidence in order to effectively justify the need for the study. Researchers should also interpret the results and evaluate what the new study adds by systemically analysing existing evidence (i.e. putting new research into context). Unfortunately, researchers fail to systematically review the current evidence when planning a new study and interpreting results.

Method The Evidence-Based Research Network (EBR network: ebrnetwork.org) was created in 2014 to promote evidencebased research - the use of prior research in a systematic and transparent way to inform a new study so that it answers the questions that matter in a valid, efficient and accessible manner. In April 2018, the Evidence-Based Research Network obtained funding from the European Cooperation in Science and Technology (COST) to create the EVidence-Based RESearch (EVBRES). COST is an organization that provides funding for researchers to create a network (called COST Action) that organizes meetings (e.g. workshops, conferences), support short term scientific visits, develop training schools, and carry out dissemination activities. EVBRES (evbres.eu), COST Action Network (CA-17117), is establishing an international network to encourage researchers and other stakeholders such as patients, ethics committee members, funders, and journal editors to use an EBR approach when conducting or supporting research.

Results EVBRES officially commenced in October 2018 with participations from researchers in more than 35 European COST Action member countries, in addition, more than 10 international partner countries were also involved. We held the first EVBRES workshop at Bergen, Norway in February 2019 and organized four working groups to carry out the mission of EVBRES. Working Group 1 will describe key stakeholders' role, such as ethic committees, funding agencies, journals and patient groups, in solidifying the evidence-based research approach. Working Group 2 will develop and organize activities aimed at educating researchers on how to systematically incorporate existing evidence when preparing new 